\title{
SURVEY ON USAGE OF MEDICINAL PLANTS: A CASE FROM CHITWAN DISTRICT OF NEPAL
}

\author{
A. Joshi ${ }^{*}$ D. Kalauni and S. Bhattarai \\ Faculty of Agriculture, Agriculture and Forestry University, Rampur, Chitwan, Nepal
}

\begin{abstract}
The aim of this study was to know the commonly available medicinal plants and to document their usages. Study was carried out around periphery of 'Gyaneswor Community Forest' of Bharatpur-16 of Chitwan district of Nepal. Altogether, forty household were selected by random sampling, and key informant interview was carried out with community forest personnel's and leading farmers. Most of the respondents of Bharatpur-16 were found to be dependent on medicinal plants for their primary health care. Because of no side effect, easy availability and cost effectiveness of medicinal plants, most people were found satisfied using it. However, the use of and preference for medicinal plant was found limited to minor diseases only. The findings of this study revealed that there are many medicinal plants in our periphery that can be used as an alternative for allopathic medicines, but they need to be systematically managed and conserved.
\end{abstract}

Keywords: Allopathic; Ayurvedic; Cultivation; Community forest; Medicine; Processing

\section{INTRODUCTION}

Nepal consists of vast biological diversity. It is ranked as $31^{\text {st }}$ richest country in the world, in terms of biodiversity and 10th richest country in the Asia region (MoAD, 2017). Despite of its small coverage area in world map, the unique and rich geography, ecology and climatic condition is attributed due to the wide altitudinal range that measures from about $60 \mathrm{~m}$ in plains to $8848 \mathrm{~m}$ to the top of the world. MoAD (2017) reported that Nepal is blessed with 12 of 867 global terrestrial ecoregions, eight climatic zones (ranging from tropical to nival) and a total of 118 ecosystems. Similarly, National herbarium and plant laboratories, Kathmandu document 1, 50,000 specimens of plants.

Among which, large number of plants were regarded to have pharmacologically active ingredients. About 700 of the total plant species reported constitutes medicinal properties, of which 238 plants species have been chemically tested for their medicinal importance.

\footnotetext{
*Corresponding Author: arati.joshi.9638@gmail.com, kalauni.dharmendra@gmail.com
} 
Several authors gave different report about the medicinal plants available in Nepal. Pandey (1961) for the first time reported 73 medicinal and aromatic plants (MAPs). Then, Department of Medicinal Plants (DMP, 1970) reported 483 species; Malla and Shakya (1984) mentioned 630 species, Manandhar (2002) reported 1002 species, Shrestha et al. (2002) reported 1614 species, Baral and Kurmi (2006) reported 1792 species, Ghimire et al. (2008) revealed a total of 1950 species and recently Rokaya et al. (2012) report 1792 to 2331 plants as potential medicinal and aromatic plant among 6653 species of angiosperm plant.

The commonly available plants are considered to have high value in terms of medicinal and pharmacological importance. The World Health Organization (WHO) (2002) mentioned that about $25 \%$ of modern medicines are developed from plants sources used traditionally. World Health Organization recorded approximately over 21,000 plant species for their medicinal uses throughout the world. Also, WHO (2011) estimate that $65-80 \%$ of population from developing countries uses medicinal and aromatic plants as remedies. Similarly, the trend of using commonly available plants for minor diseases is a usual practice in Nepal. In some part of Nepal, peoples are also found to be fully dependent on plants products for major diseases and health problems like snake bite, diabetes, stone and other. Manandhar(2002) reported that medicinal plants are responsible for maintaining the health of $70-80 \%$ population of Nepal. Also, $\operatorname{MoAD}(2017)$ revealed that rural people in Nepal use at least 1,463 species of herbal medicinal plants.

Chitwan, an inner valley of Province No. 3 is one of the potential areas for establishment of native tropical medicinal and aromatic plants. Shukla (2015) and Malla (1994), reported that the geo-physical and ecological factors of Chitwan district is suitable for the development of rich and varied vegetation and it accommodate, most of the tropical medicinal plant species. Community forest is becoming the best conservation strategy for the vivid bio-diversity. Therefore, a survey study was carried out around the periphery of the 'Gyneshwor Community Forest' of Bharatpur-16 to find out the commonly available medicinal plant and their usage in daily life of people living there.

\section{METHODOLOGY}

\section{Study area}

An ethno-botanical survey was carried out in Bharatpur-16, of Chitwan district. Chitwan is an inner valley region that lies in the longitudes of $83^{\circ} 54^{\prime} 45^{\prime}$ 'to $84^{\circ} 48^{\prime} 15^{\prime \prime} \mathrm{E}$ and latitudes of $27^{\circ} 21^{\prime} 45^{\prime \prime}$ to $27^{\circ} 52^{\prime} 30^{\prime \prime} \mathrm{N}$ (Google Earth, 2018).Elevation of the district ranges from $244 \mathrm{~m}$ to $1945 \mathrm{~m}$ (DDC, 2014 ). It is considered to be one of the potential districts to grow medicinal plant. Thegeophysical and ecological factors of Chitwan are suitable for the development of rich and varied vegetation and it accommodate, most of the tropical medicinal plant species(Shukla, 2015; Malla, 1994). Our study area, Bharatpur-16, Brahampur is 
located in northwest part of Chitwan district. It is $8 \mathrm{~km}$ away from Narayanghat, the main market hub of the district. A community forest namely 'Gyneshwor Samudayik Ban' is situated in the study area which covers a total area of 280 hectare (Munteanu, 2010). The forest is enriched with different kind of pharmaceutically important medicinal plants like Kurilo (Asparagus racemosus) Wild. The community forest provides the user group an opportunity to collect the high value medicinal plants from forest at high time to utilize them for various purpose such as to treat the disease, etc.

\section{Research Design}

Survey was carried out during Jan-Feb, 2018 in Gyneshwor Community Forest periphery. About forty community forest user households around the forest periphery were selected randomly and a scheduled interview was carried out. Key informant survey (KIS) was carried out with the personnel of community forest and leading medicinal plant cultivator/farmer. Similarly, for secondary data collection several online open access peer reviewed national and international journals, reports, conference proceedings, related website and technical bulletins were reviewed. Also the publication of District Agriculture Development Office Chitwan (DADO), District Forest Office (DFO) Chitwan, and other local INGO and NGOs were studied. The obtained data were entered in the Microsoft Excel 2010 and IBM Statistical Package for the Social Sciences (SPSS) for descriptive analysis. Graphs are generated through both SPSS and Microsoft-Excel 2007.

\section{RESULTS AND FINDINGS}

On the basis of information collected, the followings findings and discussion are presented in different table, diagrams, figures and graphs and interpreted them accordingly:

\section{Demographic features}

A total of 40 informants (18 males and 22 females) aged between 24 to 75 were interviewed in which $45 \%$ respondent were male and $55 \%$ were female. The average age of informants was 46 year old. Illiteracy rate was found $37.5 \%$ and the literacy rate was $67.5 \%$. Studies showed that majority of peoples in the community periphery were involved in agriculture, with total of $75 \%$ farmers, $17.5 \%$ service holder and $7.5 \%$ entrepreneur. About $85 \%$ of the population was found to grow medicinal plants in their kitchen garden and $87.5 \%$ population was involved in collection and marketing of medicinal plants from the community forest (Field Survey, 2018).

\section{Preference for Medicinal Plant}

Majority of respondent (90\%) preferred medicinal plant for the treatment of disease over processed medicine. However, all of them were not found to be involved in preparing medicines for treatment of diseases and disorders. Only $77.5 \%$ population have prepared medicine using medicinal plant for curing cuts, burns and diseases, 
while $22.5 \%$ population had never used medicinal plant for preparing medicine (Figure-2).

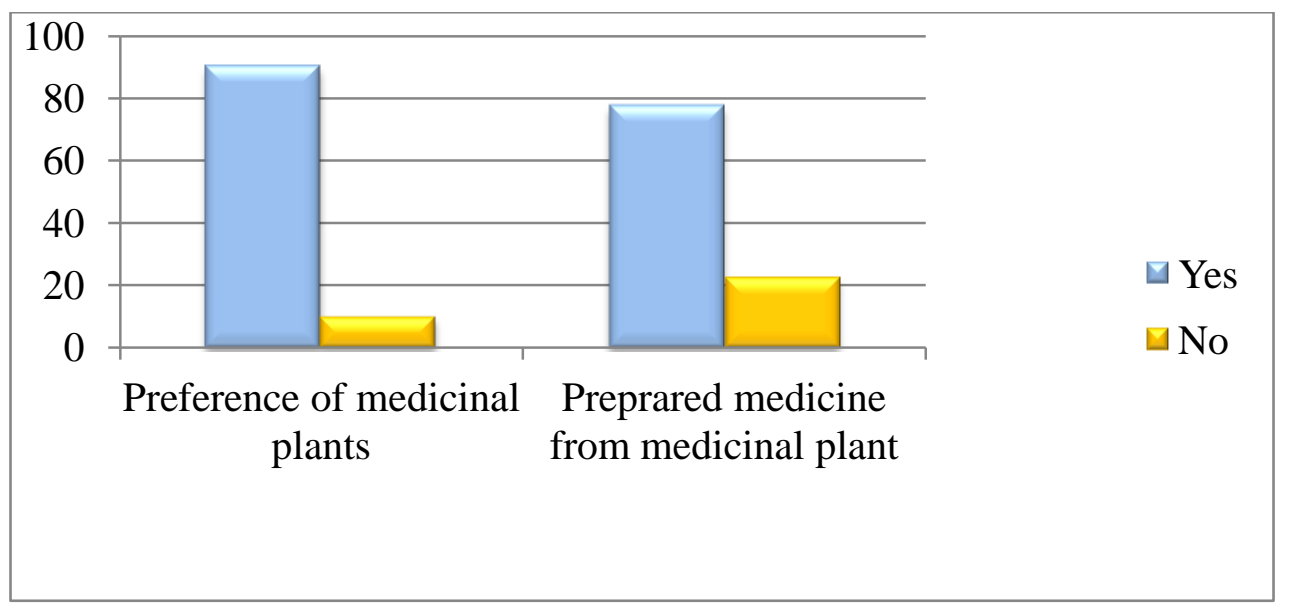

Figure 2. Respondent Preference for Medicinal Plant

\section{Preference for Ayurvedic Medicines (Allopathic Medicine)}

For minor diseases, like common cold, minor allergies, burns, cuts, cough and others $82.5 \%$ population preferred to use Ayurvedic medicines and the rest $17.5 \%$ population preferred to use allopathic medicine. However, for treatment of major diseases like cancer, stone, diabetes only $10 \%$ preferred to use Ayurvedic medicine and $90 \%$ population preferred to use allopathic medicine (Fig. 3).

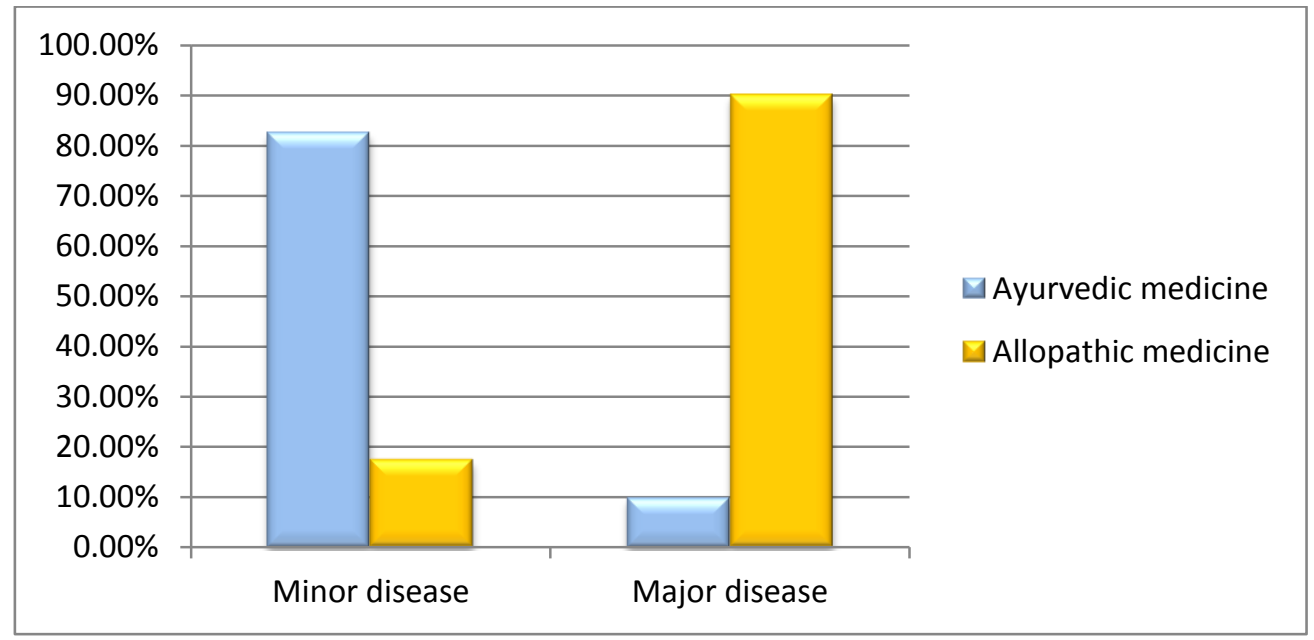

Figure 3.Percentage of respondent preferring ayurvedic medicine over allopathic medicine for major and minor diseases 


\section{Taxonomic Diversity in the community and Plants Used for medicine}

Forty-four medicinal plant species belonging to 29 families were found to be used by the local community. Among which 14 plant species were used to treat stomach disorders like stomachache, diarrhea, dysentery, constipation and others, 9 plant species were used for wound healing, 8 plant species were used for treatment of cold, 7 plant species were found to be used for cough, 3plant species were found to be used for fever. Similarly, 4plant species were used to recover diabetes, 3 plant species were used to maintain blood pressure, 3 species were used for treating stone, 3 plant species were used as blood purifier, 3 plant species were used for menstrual problem, 2 species were used for improving lactation of females after pregnancy, 2 species for toothache and 1 species was found to be used for treating cancer.

\section{Satisfaction Gained by using Medicinal Plants}

The medicinal plant users were found satisfied with its effect to a great extent. Majority of respondent about $62.5 \%$ reported a complete healing effect of medicinal plant for treatment of minor disease. However, $35 \%$ of the respondent felt moderate healing effect and $2.5 \%$ respondent felt appreciable effect of medicinal plant for curing minor diseases (Figure 4). Similarly, for major diseases, $20 \%$ of the respondent reported to feel a moderate healing effect with the use of medicinal plants.About $32.5 \%$ population was found to feel appreciable healing effect and 47.5\% population was found to feel negligible healing effect. Almost the entire respondent about $97.5 \%$ said that they never noticed any side effect of medicinal plants. However, only $2.5 \%$ of respondent reported to the side effect of medicinal plant (Figure 5). Respondent told that a medicinal plant, locally called Dahikamala (Rhustyphina L.) was found to lower the blood pressure, if taken in excess amount.

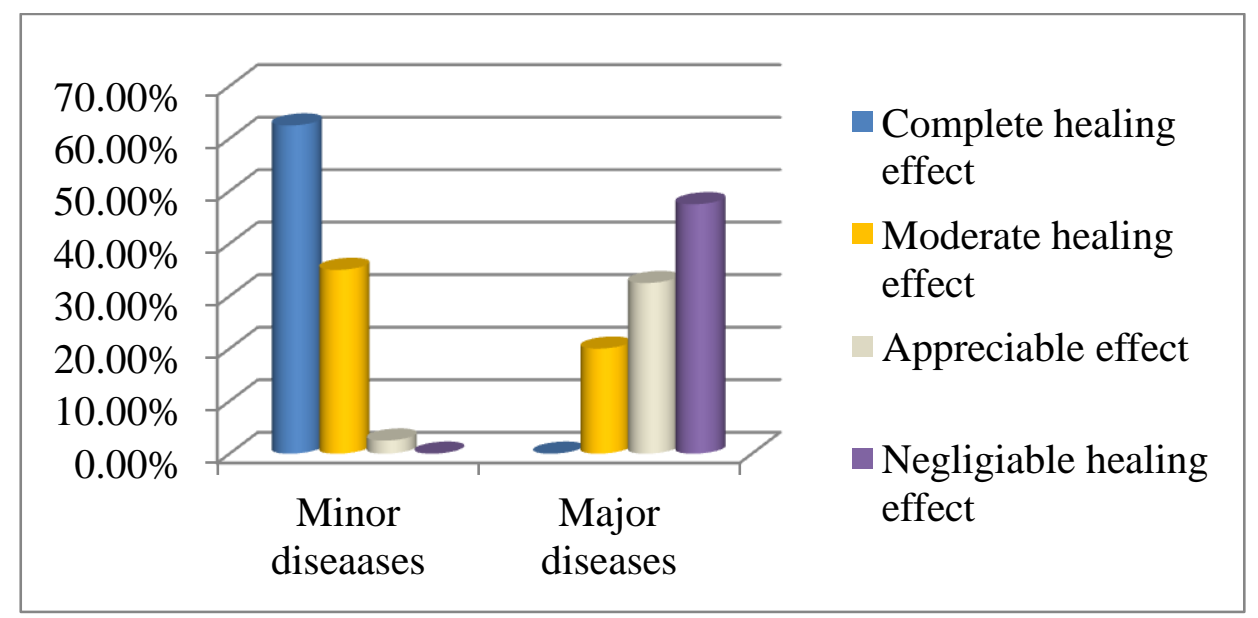

Fig. 4. Various category of healing effect among respondent 


\section{Side effect among respondent}

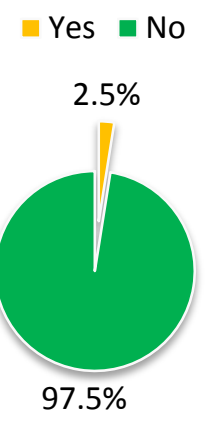

Figure 5. Percentage of Respondent Experiencing the Side Effect of Medicinal Plant

The usages of commonly available medicinal plants are described in the table below:

Table 1. Commonly Cultivated Medicinal Plant in Study Area

\begin{tabular}{|c|c|c|c|c|c|c|}
\hline $\begin{array}{l}\text { S. } \\
\text { N. }\end{array}$ & $\begin{array}{l}\text { Common } \\
\text { Name }\end{array}$ & $\begin{array}{l}\text { Biological } \\
\text { Name }\end{array}$ & Family & Used for & $\begin{array}{l}\text { Used } \\
\text { parts }\end{array}$ & $\begin{array}{l}\text { Propagated } \\
\text { by }\end{array}$ \\
\hline 01. & $\begin{array}{l}\text { Besar } \\
\text { (Turmeric) }\end{array}$ & $\begin{array}{l}\text { Curcuma } \\
\text { longa (L.) }\end{array}$ & Zingebeaceae & $\begin{array}{l}\text { Rhizome powder is boiled with } \\
\text { water for treating common cold } \\
\text { Paste is used in inflammation, } \\
\text { wound healing }\end{array}$ & $\begin{array}{l}\text { Rhizor } \\
\text { e }\end{array}$ & Rhizome \\
\hline 02. & Tulsi & $\begin{array}{l}\text { Ocimum } \\
\text { sanctum (L.) }\end{array}$ & Lamiaceae & $\begin{array}{l}\text { Leaf decoction used for cough } \\
\text { and cold, respiratory disorders } \\
\text { and reduces stress }\end{array}$ & $\begin{array}{l}\text { Whole } \\
\text { plant }\end{array}$ & Seed \\
\hline 03. & Neem & $\begin{array}{l}\text { Azadirachtain } \\
\text { dicaA. Juss. }\end{array}$ & Meliaceae & $\begin{array}{l}\text { Leaf bark decoction used to cure } \\
\text { fever, leaf extract used for skin } \\
\text { diseases and as botanical } \\
\text { insecticide }\end{array}$ & $\begin{array}{l}\text { Leaf, } \\
\text { bark }\end{array}$ & $\begin{array}{l}\text { Seed, } \\
\text { Cutting }\end{array}$ \\
\hline 04. & $\begin{array}{l}\text { Ghiukumari, } \\
\text { Aloe }\end{array}$ & Aloe vera $(\mathrm{L})$. & Liliaceae & $\begin{array}{l}\text { Leaf gel used to treat cut, } \\
\text { wounds and burns } \\
\text { Used as cosmetic }\end{array}$ & Leaf & Leaf, offset \\
\hline 05. & $\begin{array}{l}\text { Sarpagandha } \\
\text {, Snake plant }\end{array}$ & $\begin{array}{l}\text { Rauvolfiaserp } \\
\text { entina } \\
\text { (L.) Benth ex } \\
\text { Kurz }\end{array}$ & Apocynaceae & $\begin{array}{l}\text { Treatment of high blood } \\
\text { pressure. Root powder is } \\
\text { applied on area of snake or } \\
\text { insect bite. Root decoction used } \\
\text { for uterine contraction }\end{array}$ & $\begin{array}{l}\text { Roots, } \\
\text { leaves }\end{array}$ & $\begin{array}{l}\text { Seed, stem } \\
\text { cutting, } \\
\text { Root } \\
\text { cutting }\end{array}$ \\
\hline 06. & $\begin{array}{l}\text { Kurilo, } \\
\text { Asparagus }\end{array}$ & $\begin{array}{l}\text { Asparagus } \\
\text { racemosusWi } \\
\text { lld. }\end{array}$ & Asparagaceae & $\begin{array}{l}\text { Powdered root is considered as } \\
\text { tonic. Consumption of roots } \\
\text { supports milk production in } \\
\text { females. Also considered } \\
\text { beneficial for treating kidney } \\
\text { stone }\end{array}$ & $\begin{array}{l}\text { Root, } \\
\text { tuber, } \\
\text { stem, } \\
\text { fruit }\end{array}$ & $\begin{array}{l}\text { Seed, } \\
\text { Cutting }\end{array}$ \\
\hline
\end{tabular}




\begin{tabular}{|c|c|c|c|c|c|c|}
\hline $\begin{array}{l}\text { S. } \\
\text { N. }\end{array}$ & $\begin{array}{l}\text { Common } \\
\text { Name }\end{array}$ & $\begin{array}{l}\text { Biological } \\
\text { Name }\end{array}$ & Family & Used for & $\begin{array}{l}\text { Used } \\
\text { parts }\end{array}$ & $\begin{array}{c}\text { Propagated } \\
\text { by }\end{array}$ \\
\hline 07. & $\begin{array}{l}\text { Dhaniya, } \\
\text { Cilantro }\end{array}$ & $\begin{array}{l}\text { Coriandrums } \\
\text { ativum }(\mathrm{L} .)\end{array}$ & Umbelliferae & $\begin{array}{l}\text { Leaves and } \\
\text { appetizer. } \\
\text { digestion. }\end{array}$ & $\begin{array}{l}\text { Whole } \\
\text { plant }\end{array}$ & Seed \\
\hline 09. & $\begin{array}{l}\text { Aduwa, } \\
\text { Ginger }\end{array}$ & $\begin{array}{l}\text { Zingiberoffici } \\
\text { nale Roscoe }\end{array}$ & Zingeberaceae & $\begin{array}{l}\text { Rhizome water is used for cold, } \\
\text { throat sore } \\
\text { Used for stomach disorder }\end{array}$ & $\begin{array}{l}\text { Rhizom } \\
\text { e }\end{array}$ & Rhizome \\
\hline 10. & $\begin{array}{l}\text { Pipla, Long } \\
\text { Pepper }\end{array}$ & $\begin{array}{l}\text { Piper longum } \\
\text { (L.) }\end{array}$ & Piperaceae & $\begin{array}{l}\text { Powdered leaf along with honey } \\
\text { is useful for cold, cough } \\
\text { Infusion of root used for } \\
\text { stomachache, bronchitis }\end{array}$ & $\begin{array}{l}\text { Root, } \\
\text { Leaf }\end{array}$ & $\begin{array}{l}\text { Seed, stem } \\
\text { cutting }\end{array}$ \\
\hline 11. & $\begin{array}{l}\text { Amala, } \\
\text { Indian } \\
\text { gooseberry }\end{array}$ & $\begin{array}{l}\text { Phyllanthusof } \\
\text { ficinalis(L.) }\end{array}$ & Phyllanthaceae & $\begin{array}{l}\text { Juice of fruit or fresh fruit is } \\
\text { consumed to reduce blood sugar } \\
\text { Barks and fruits are useful for } \\
\text { diarrhea, dysentery }\end{array}$ & $\begin{array}{l}\text { Fruit, } \\
\text { Barks }\end{array}$ & Cutting \\
\hline 12. & Jira, Cumin & $\begin{array}{l}\text { Cuminuncymi } \\
\text { num(L.) }\end{array}$ & Umbelliferae & $\begin{array}{l}\text { Cumin seed are used for } \\
\text { diarrhea, colic disorder, } \\
\text { bloating. } \\
\text { It is used to start menstruation } \\
\text { earlier. }\end{array}$ & Seed & Seed \\
\hline 13. & $\begin{array}{l}\text { Tejpatta, } \\
\text { Indian cassia }\end{array}$ & $\begin{array}{l}\text { Cinnamomum } \\
\text { tamala }(\text { Buch } \\
-\quad \text { Ham. }) \mathrm{T} . \\
\text { Nees\& C.H. } \\
\text { Eberm }\end{array}$ & Lauraceae & $\begin{array}{l}\text { Powder of bark is mixed with } \\
\text { honey for treating cough, } \\
\text { asthma } \\
\text { Paste of bark is applied over } \\
\text { wound, inflammation }\end{array}$ & $\begin{array}{l}\text { Leaves, } \\
\text { Barks }\end{array}$ & $\begin{array}{l}\text { Cutting, } \\
\text { Seed }\end{array}$ \\
\hline 14. & Titepati & $\begin{array}{l}\text { Artemisia } \\
\text { vulgaris (L.) }\end{array}$ & Asteraceae & $\begin{array}{l}\text { Stomach disorder, } \\
\text { Leaf paste applied on wounds, } \\
\text { cuts }\end{array}$ & $\begin{array}{l}\text { Whole } \\
\text { Plant }\end{array}$ & $\begin{array}{l}\text { Seeds and } \\
\text { basal } \\
\text { cutting }\end{array}$ \\
\hline 15. & Datura & $\begin{array}{l}\text { Datura metal } \\
\text { (L.) }\end{array}$ & l Solanaceae & $\begin{array}{l}\text { Paste of roasted leaves are } \\
\text { applied over wound } \\
\text { Leaf smoke is used to treat } \\
\text { asthma }\end{array}$ & $\begin{array}{l}\text { Leaf, } \\
\text { seed }\end{array}$ & $\begin{array}{l}\text { Seed, Root } \\
\text { cutting }\end{array}$ \\
\hline 16. & Bryophyllum & $\begin{array}{l}\text { Bryophyllump } \\
\text { innatum(Lam } \\
\text {.) Oken }\end{array}$ & Crassulaceae & $\begin{array}{l}\text { Decoction of leaves is used for } \\
\text { kidney stone, stomachache } \\
\text { Leaf juice applied for ear pain } \\
\text { Extract of aerial part treat } \\
\text { cancer } \\
\text { Root used for blood pressure }\end{array}$ & $\begin{array}{l}\text { Leaf, } \\
\text { Root }\end{array}$ & Leaf \\
\hline 17. & $\begin{array}{l}\text { Asare, Curry } \\
\text { leaf }\end{array}$ & $\begin{array}{l}\text { Murrayakoen } \\
\text { igii(L.) } \\
\text { Sprengel }\end{array}$ & Rutaceae & $\begin{array}{l}\text { Juice of leaf is used to treat } \\
\text { diarrhea } \\
\text { Paste of leaf applied in hair } \\
\text { helps hair growth } \\
\text { Juice or paste of leaf is applied } \\
\text { to cuts, burns, wound }\end{array}$ & Leaf & $\begin{array}{l}\text { Seed, Stem } \\
\text { cutting }\end{array}$ \\
\hline
\end{tabular}




\begin{tabular}{|c|c|c|c|c|c|c|}
\hline $\begin{array}{l}\text { S. } \\
\text { N. }\end{array}$ & $\begin{array}{l}\text { Common } \\
\text { Name }\end{array}$ & $\begin{array}{l}\text { Biological } \\
\text { Name }\end{array}$ & Family & Used for & $\begin{array}{l}\text { Used } \\
\text { parts }\end{array}$ & $\begin{array}{c}\text { Propagated } \\
\text { by }\end{array}$ \\
\hline \multirow[t]{3}{*}{18.} & $\begin{array}{l}\text { Methi, } \\
\text { Fenugreek }\end{array}$ & $\begin{array}{l}\text { Trigonellafoe } \\
\text { num- } \\
\text { graecum }(\mathrm{L} .)\end{array}$ & Fabaceae & $\begin{array}{l}\text { Paste of fenugreek leaf is } \\
\text { applied in hair to reduce } \\
\text { dandruff }\end{array}$ & $\begin{array}{l}\text { Leaf, } \\
\text { Seed }\end{array}$ & Seed \\
\hline & & & & $\begin{array}{l}\text { Seeds are useful for diabetic } \\
\text { patient }\end{array}$ & & \\
\hline & & & & $\begin{array}{l}\text { They improve lactation after } \\
\text { delivery }\end{array}$ & & \\
\hline \multirow[t]{2}{*}{19.} & Pudina, Mint & Mentha & Lamiaceae & Mint leaf are used for toothache & Leaf & Cutting \\
\hline & & (L.) & & $\begin{array}{l}\text { Mint leaves are added to steam } \\
\text { bath for relieving running nose }\end{array}$ & & \\
\hline
\end{tabular}

Table 2. Usage of Collected medicinal plants from the community forest

\begin{tabular}{|c|c|c|c|c|c|c|}
\hline S.N. & $\begin{array}{l}\text { MAPs } \\
\text { collected }\end{array}$ & Scientific Name & Family & Uses & Used part & Propagated by \\
\hline 01. & Abhijalo & $\begin{array}{l}\text { Drymariacordata( } \\
\text { L.) }\end{array}$ & $\begin{array}{l}\text { Caryophyllacea } \\
\text { e }\end{array}$ & $\begin{array}{l}\text { To treat gastric trouble, } \\
\text { Pneumonia }\end{array}$ & $\begin{array}{l}\text { Whole } \\
\text { plant, fresh } \\
\text { leaves }\end{array}$ & Grow wild \\
\hline 02. & Chiraito & $\begin{array}{l}\text { Swertiachirayita } \\
\text { Roxb. ex Fleming) } \\
\text { Karsten }\end{array}$ & Gentianaceae & $\begin{array}{l}\text { Increases metabolism, swiftly } \\
\text { lowers fever and constipation. }\end{array}$ & All parts & Seed \\
\hline 03. & Dahikamala & Rhustyphina(L.) & Anacardiaceae & $\begin{array}{l}\text { Berries are used as astringent } \\
\text { and blood purifier. Roots are } \\
\text { used to treat boils. }\end{array}$ & Roots & Seed \\
\hline 04. & Gandhe & $\begin{array}{l}\text { Ageratum } \\
\text { conyzoides }(\mathrm{L} .)\end{array}$ & Asteraceae & $\begin{array}{l}\text { Aqueous extracts of leaves or } \\
\text { whole plants are to treat colic, } \\
\text { colds, fevers and diarrhea. }\end{array}$ & Whole plant & Grow wild \\
\hline 05. & GhodTapre & $\begin{array}{l}\text { Centellaasiatica } \\
\text { (L.) Urban }\end{array}$ & Apiaceae & $\begin{array}{l}\text { Used for fatigue, anxiety, } \\
\text { improving memory and } \\
\text { intelligence. }\end{array}$ & Whole plant & $\begin{array}{l}\text { Grow locally } \\
\text { and wild }\end{array}$ \\
\hline 06. & Lajjawatijhar & $\begin{array}{l}\text { Mimosa } \\
\text { pudica }(\mathrm{L} .)\end{array}$ & Fabaceae & $\begin{array}{l}\text { Root is used in treatment of } \\
\text { dysentery, vaginal and uterine } \\
\text { complaints, and skin diseases. }\end{array}$ & $\begin{array}{l}\text { Root } \\
\text { leaves }\end{array}$ & $\begin{array}{l}\text { Propagated by } \\
\text { vegetative } \\
\text { means }\end{array}$ \\
\hline 07. & Pahelolahara & $\begin{array}{l}\text { Clematis } \\
\text { buchananiana (de } \\
\text { Candolle) }\end{array}$ & Ranunculaceae & $\begin{array}{l}\text { Juice of the root is used in the } \\
\text { cough and to cure peptic ulcer. }\end{array}$ & Roots & Cuttings \\
\hline 08. & Tulsi & $\begin{array}{l}\text { Ocimum sanctum } \\
\text { (L.) }\end{array}$ & Lamiaceae & $\begin{array}{l}\text { Leaf decoction used for cough } \\
\text { and cold,respiratory disorders } \\
\text { and reduces stress. }\end{array}$ & Whole plant & Seed \\
\hline 09. & $\begin{array}{l}\text { Ashwagandh } \\
\text { a }\end{array}$ & $\begin{array}{l}\text { Withaniasomnifer } \\
a \\
\text { (L.) Dunal }\end{array}$ & Solanaceae & $\begin{array}{l}\text { It is used to lower blood } \\
\text { pressure and to increase } \\
\text { hemoglobin and hair melanin. }\end{array}$ & Roots & Seed \\
\hline 10. & Gurjo & $\begin{array}{l}\text { Tinosporacordifoli } \\
a \\
\text { (Thunb.) Miers }\end{array}$ & $\begin{array}{l}\text { Menispermacea } \\
\text { e }\end{array}$ & $\begin{array}{l}\text { Used in different metabolic } \\
\text { disorders and gases }\end{array}$ & Whole part & Cutting \\
\hline
\end{tabular}




\begin{tabular}{|c|c|c|c|c|c|c|}
\hline S.N. & $\begin{array}{l}\text { MAPs } \\
\text { collected }\end{array}$ & Scientific Name & Family & Uses & Used part & Propagated by \\
\hline 11. & Jwano & $\begin{array}{l}\text { Trachyspermuma } \\
\text { mmi Sprague }\end{array}$ & Apiaceae & $\begin{array}{l}\text { Fruits are used to cure } \\
\text { abdominal tumors, piles, lack } \\
\text { of appetite. }\end{array}$ & Seeds & Seeds \\
\hline 12. & Kutiko & $\begin{array}{l}\text { Neopicrorhizascro } \\
\text { phulariiflora }(\text { Penn } \\
\text { ell) Hong }\end{array}$ & $\begin{array}{l}\text { Scrophulariace } \\
\text { aae }\end{array}$ & $\begin{array}{l}\text { Used for indigestion, fever, } \\
\text { jaundice, etc }\end{array}$ & Rhizome & $\begin{array}{l}\text { Rhizome and } \\
\text { Root }\end{array}$ \\
\hline 13. & Neem & $\begin{array}{l}\text { Azadirachtaindica } \\
\text { A.Juss. }\end{array}$ & Meliaceae & $\begin{array}{l}\text { Leaf bark decoction used to } \\
\text { cure fever, leaf extract used for } \\
\text { skin diseases and as botanical } \\
\text { insecticide }\end{array}$ & Leaf, bark & $\begin{array}{l}\text { Seed } \\
\text { cuttings }\end{array}$ \\
\hline \multirow[t]{2}{*}{14.} & Mint & Menthasp.(L.) & Lamiaceae & $\begin{array}{l}\text { Mint leaf are used for } \\
\text { toothache }\end{array}$ & $\begin{array}{l}\text { Leaf } \\
\text { stem }\end{array}$ & Cutting \\
\hline & & & & $\begin{array}{l}\text { Mint leaves are added to steam } \\
\text { bath for relieving running nose }\end{array}$ & & \\
\hline 15. & Hadchur & Viscum album (L.) & Viscaceae & $\begin{array}{l}\text { To lower blood pressure and } \\
\text { heart rate, and induce sleeping. }\end{array}$ & $\begin{array}{l}\text { All parts of } \\
\text { the plant }\end{array}$ & $\begin{array}{l}\text { Vegetative or } \\
\text { seed propagati } \\
\text { on }\end{array}$ \\
\hline 16. & $\begin{array}{l}\text { Raktachanda } \\
\mathrm{n}\end{array}$ & $\begin{array}{l}\text { Pterocarpussantal } \\
\text { inus(L.f.) Kuntze }\end{array}$ & Fabaceae & $\begin{array}{l}\text { Heart-wood are for heart } \\
\text { diseases, blood purifier, } \\
\text { headache and skin diseases. }\end{array}$ & Heart wood & Seeds \\
\hline 17. & $\begin{array}{l}\text { Rato } \\
\text { Mushroom }\end{array}$ & $\begin{array}{l}\text { Amanita } \\
\text { muscaria }(\mathrm{L} .) \text { Lam. }\end{array}$ & Amantiaceae & Against diarrhea & Whole plant & Spores \\
\hline 18. & Bhang & $\begin{array}{l}\text { Cannabis } \\
\text { indica }(\mathrm{L} .)\end{array}$ & Cannabaceae & $\begin{array}{l}\text { Useful in combating menstrual } \\
\text { discomfort and reproductive } \\
\text { problems. }\end{array}$ & Leaves & Seed \\
\hline 20. & $\begin{array}{l}\text { Tejpatta/ } \\
\text { Indian Cassia }\end{array}$ & $\begin{array}{l}\text { Cinnamomumtama } \\
\text { la(Buch.- } \\
\text { Ham.) T.Nees \& C } \\
\text {.H.Eberm }\end{array}$ & Lauraceae & $\begin{array}{l}\text { Powder of bark is mixed with } \\
\text { honey for treating cough, } \\
\text { asthma } \\
\text { Paste of bark is applied over } \\
\text { wound, inflammation }\end{array}$ & $\begin{array}{l}\text { Leaves, } \\
\text { Barks }\end{array}$ & Cutting, Seed \\
\hline 21. & Jamun & $\begin{array}{l}\text { Syzygiumcumini }(\mathrm{L} \\
\text {.) Skeels }\end{array}$ & Myrtaceae & $\begin{array}{l}\text { Leaves are used for treatment } \\
\text { of diabetes, and menstrual } \\
\text { problems. }\end{array}$ & $\begin{array}{l}\text { Fruits, } \\
\text { Seeds, } \\
\text { Leaves, } \\
\text { bark }\end{array}$ & $\begin{array}{l}\text { Air layering } \\
\text { and softwood } \\
\text { grafting }\end{array}$ \\
\hline 22. & $\begin{array}{l}\text { Nerbanshe/ } \\
\text { Nirmanse }\end{array}$ & $\begin{array}{l}\text { Delphinium } \\
\text { denudatumWall }\end{array}$ & Ranunculaceae & $\begin{array}{l}\text { Against sugar patients, Relieve } \\
\text { stomach pain and it is good } \\
\text { blood purifier. }\end{array}$ & Root, Seed & $\begin{array}{l}\text { Seeds are } \\
\text { propagating } \\
\text { materials }\end{array}$ \\
\hline 23. & Chutro & $\begin{array}{l}\text { BerberisaristataD } \\
\text { C }\end{array}$ & Berberidaceae & $\begin{array}{l}\text { Roots are used for Poisonous } \\
\text { remedy }\end{array}$ & $\begin{array}{l}\text { Roots, } \\
\text { Fruits,Stem } \\
\text { and Leaves }\end{array}$ & Seed \\
\hline 24. & Dalchini & $\begin{array}{l}\text { Cinnamomumveru } \\
m J . P r e s 1\end{array}$ & Lauraceae & $\begin{array}{l}\text { Paste of this herb is used to } \\
\text { reduce pain, inflammation and } \\
\text { eye related disorders. }\end{array}$ & $\begin{array}{l}\text { Bark, } \\
\text { Leaves and } \\
\text { berry fruit }\end{array}$ & $\begin{array}{l}\text { Cuttings, } \\
\text { Layering and } \\
\text { by dividing } \\
\text { the root ball }\end{array}$ \\
\hline 25. & Gheukumari & $\begin{array}{ll}\text { Aloe } & \text { vera } \\
\text { (L.) Burm.f. } & \end{array}$ & Liliaceae & $\begin{array}{l}\text { Leaf gel used to treat cut, } \\
\text { wounds and burns }\end{array}$ & Leaf & Leaf, offset \\
\hline & & & & Used as cosmetic & & \\
\hline
\end{tabular}




\begin{tabular}{|c|c|c|c|c|c|c|}
\hline S.N. & $\begin{array}{l}\text { MAPs } \\
\text { collected }\end{array}$ & Scientific Name & Family & Uses & Used part & Propagated by \\
\hline 26. & Kurilo & $\begin{array}{l}\text { Asparagus } \\
\text { racemosusWilld. }\end{array}$ & Asparagaceae & $\begin{array}{l}\text { Powdered root is considered as } \\
\text { tonic } \\
\text { Consumption of roots supports } \\
\text { milk production in females } \\
\text { Also considered beneficial for } \\
\text { treating kidney stone }\end{array}$ & $\begin{array}{l}\text { Root, tuber, } \\
\text { stem, fruit }\end{array}$ & Seed, Cutting \\
\hline 27. & Lude & $-? ? ? ? ?$ & - & $\begin{array}{l}\text { Root is used in the form of } \\
\text { juice, paste, decoction to treat } \\
\text { intrinsic hemorrhage, diarrhea, } \\
\text { hoarseness of voice, cough. }\end{array}$ & & - \\
\hline 28. & Pipla & Piper longum(L.) & Piperaceae & $\begin{array}{l}\text { Powdered leaf along with } \\
\text { honey is useful for cold, cough } \\
\text { Infusion of root used for } \\
\text { stomachache, bronchitis }\end{array}$ & Root, Leaf & $\begin{array}{l}\text { Seed, } \\
\text { cutting }\end{array}$ \\
\hline 29. & Sarpagandha & $\begin{array}{l}\text { Rauvolfia } \\
\text { serpentine } \\
\text { (L.) Benth. ex Kur } \\
\text { z }\end{array}$ & Apocynaceae & $\begin{array}{l}\text { Treatment of high blood } \\
\text { pressure } \\
\text { Root powder is applied on area } \\
\text { of snake or insect bite } \\
\text { Root decoction used for uterine } \\
\text { contraction }\end{array}$ & $\begin{array}{l}\text { Roots, } \\
\text { leaves }\end{array}$ & $\begin{array}{ll}\text { Seed, } & \text { stem } \\
\text { cutting, } & \text { Root } \\
\text { cutting } & \end{array}$ \\
\hline 30. & Sotuwa & $\begin{array}{l}\text { Paris } \\
\text { polyphyllaSm. }\end{array}$ & Melanthiaceae & $\begin{array}{l}\text { Used against any poisonous } \\
\text { bite, burn, cut or injury. }\end{array}$ & Rhizome & Seed \\
\hline 31. & Rudhilo & $\begin{array}{l}\text { Pogostemonbengh } \\
\text { alensis(Burm.f.) } \\
\text { Kuntze }\end{array}$ & Lamiaceae & $\begin{array}{l}\text { Leaves are used to clean } \\
\text { wounds and promote their } \\
\text { healing. Used for lactating } \\
\text { cows }\end{array}$ & Leaf, Root & Found wild \\
\hline 32. & Harro & $\begin{array}{l}\text { Terminaliachebula } \\
\text { Retz }\end{array}$ & Combretaceae & $\begin{array}{l}\text { Paste of fruits is used to clean } \\
\text { wounds, to provide relief to } \\
\text { eyelids in cases of } \\
\text { conjunctivitis. }\end{array}$ & Bark, Fruit & Cutting \\
\hline 33. & Bojho & $\begin{array}{l}\text { AcoruscalamusLin } \\
\text { n. calamus }\end{array}$ & Acoraceae & $\begin{array}{l}\text { Used for cold and cough, cure } \\
\text { piles, loss of appetite, urinary } \\
\text { stones, and to improve } \\
\text { memory. }\end{array}$ & $\begin{array}{l}\text { Rhizome, } \\
\text { leaf }\end{array}$ & Rhizome \\
\hline
\end{tabular}

\section{DISCUSSION}

The user groups of community forest were found have good knowledge of medicinal plants and their usage. They used 44 medicinal plants for treating 62 ailments. A single plant was found to have multiple benefits. A similar result for the usages of medicinal plant was reported by Singh et al., (2012). The authors reported 66 medicinal plant species to treat various disorders, including gastro-intestinal disorder and dermatological disorders majorly in Rupandehi district. Similarly, Uprety et al. (2010) found 56 plant species in Rasuwa district, which were used for treating gastrointestinal problems, fever and headache. Bhattarai et al., (2006) found 91 locally used medicinal plants which were used to treat 93 ailments in Manang district. 
Mostly, the farmers and elderly people prepared medicine from medicinal plant. The respondent who were involved in job or business were less involved in the preparation of medicines from medicinal plant. They mostly used the allopathic medicines. However, most of the respondent, irrespective of their occupation preferred ayurvedic medicines for the treatment of minor diseases. Since the user groups have not noticed much side effect of medicines prepared from medicinal plants therefore, they use such plant instantly for treatment. Similar results were reported by Joshi et al. (2011). The authors reported that the use of medicinal plant was limited to minor health related problems like cuts, wounds, gastro-intestinal problems, fever, headache, cough and others in Macchegaun, Kathmandu.

Our finding revealed that the majority of the people preferred allopathic medicines for the treatment of major diseases. It is because of the slow acting effect of ayurvedic medicine. Only a few peoples prefer to use ayurvedic medicine even for the major disease. The respondent explained that they have experienced positive effect of ayurvedic medicine for curing major diseases. They believed that continuous use of medicinal plants in proper way can cure the so called medically incurable diseases like cancer. Unlike the processed medicine, the medicinal plants gave a natural healing without the worries of any side effect. Gewali (2008) also reported that the folk medicines have no or little side effects. Similarly, Koirala and Khaniya (2009) reported that though the effect of medicines prepared from medicinal plants is slow, but medicines do not have any side effects. Further, they reported that such medicines are proved to be successful in treatment of many major diseases like hepatitis (any type), multiple sclerosis, any type of arthritis, many cancerous diseases like breast cancer, prostate cancer, and also many cases of tumor and cysts, metastatic conditions, immunity, etc. In our finding, we found that peoples used the medicinal plants to cure cancer, kidney stone, uterine stone, asthma, piles, conjunctivitis, jaundice, pneumonia, poisonous bite and others (Table 1 and 2). Further, common availability of medicinal plants in their kitchen garden, field and community forest makes its use cost effective over the allopathic medicines. Koirala and Khaniya (2009) also reported that the effect of medicinal plant is not only observed in human health but also in better living standard and income. Harvesting and gathering of the medicinal and aromatic plants provide subsistence living income.

\section{CONCLUSION}

The peoples living around the community forest periphery were found to use the medicinal plants for curing of minor and major diseases. Less side effect, easy availability and cost effectiveness of the medicinal plants make it a preference over allopathic medicines, for most of the rural household. Peoples were found to be satisfied by the effectiveness of medicinal plants. However, the usage of medicinal plants completely grounds on the indigenous knowledge and traditional beliefs of the peoples. Such knowledge differs from community to community and also with religion, culture and geography. 
Therefore, a chemical screening and evaluation of such medicinal plants must be carried out so as to identify the bioactive compound for further preparation of drugs. It is suggested that providing training on cultivation and identification of the important medicinal and aromatic plants would be beneficial to commercialize, conserve and utilize the commonly available medicinal plants.

\section{REFERENCES}

Baral, S.R., and Kurmi, P.P. (2006). A compendium of medicinal plants in Nepal., pp. 450451. Pub.: MrsRachana Sharma, MaijuBahal, Chabhil, Kathmandu, Nepal.

Bhattarai, S., Chaudhary, R.P., and Taylor, R.S. (2006). Ethnomedicinal plants used by the people of Manang district, Central Nepal. Journal of Ethnobiology Ethnomedicine 2: 41 doi: 10.1186/1746-4269-2-41.

DDC. (2014). District Climate And Energy Plan Chitwan District. Chitwan: District Development Committee.

DMP. (1970). Medicinal plant of Nepal: Bulletin no. 3. Kathmandu: Department of Medicinal Plants.

Gewali, M.B. (2008). Aspects of Traditional Medicine Aspects of Traditional Medicine in Nepal ed. Suresh Awale. Toyama, Japan: Institute of Natural Medicine, University of Toyama.

Ghimire, S., Sapkota, I., Oli, B., and Parajuli, R. (2008). Nontimber forest products of Nepal Himalaya: Database of some important species found in the mountain protected areas andsurrounding regions. Kathmandu: WWF Nepal.

Joshi, K., Joshi, R., and Joshi, A. (2011). Indigenous knowledge and uses of medicinal plants in Macchegaun, Nepal. Indian Journal of Traditional Knowledge, 10(2), 281-286.

Koirala, R.R., and Khaniya, B.N. (2009). Present Status of Traditional Medicines and Medicinal \& Aromatic Plants Related Resources \& Organizations in Nepal. Kathmandu, Nepal: Nepal Health Research Council.

Malla, S. (1994). Medicinal Herbs in Bagmati Zone. Lalitpur, Nepal: ICIMOD.

Mall, S. and Shakya, P. (1984). Medicinal Plants. Nepal Nature Paradise, pp. 261-297. Bangkok, Thailand: Majpuria TC (eds.) White Lotous Co Ltd.

Manandhar, N. (2002). Plants and People of Nepal. Oregon, USA: Timber Press Portland.

MoAD. (2017). The State of Nepal's Biodiversity for Food and Agriculture. Kathmandu: Ministry of Agriculture Development.

Munteanu, A.R. (2010). The potential of medicinal plant cultivation as an endogenous development strategy-A study based in Chitwan District, Nepal. retrieved from http://www. scribd. com/doc/41611458/The-Potential-of-Medicinal-Plant-Cultivationas-an-Endogenous-Development-Strategy-AR-Munteanu-ERM-Thesis.

Pandey, P. (1961). Distribution of medicinal plants of Nepal. Symposium on Medicinal Plants, pp. 15-61. Ceylon.

Rokaya, M.B., Münzbergová, Z., Shrestha, M.R., and Timsina, B. (2012). Distribution Patterns of Medicinal Plants along an Elevational Gradient in Central Himalaya, Nepal. Journal of Mountain Science, 201-213. 
Shrestha, K.K., Tiwari, N.N., and Ghimire, S.K. (2002). Medicinal and aromatic plants database of Nepal. In: Watanabe T, Takano A, Bista MS, Saiju HK (eds.), Proceeding of Nepal-Japan Joint Symposium on Conservation and Utilization of Himalayan Medicinal Plant Resources.

Shukla, A. (2015). Medicinal and Aromatic Plants (MAPs) Stakeholders Directory. Kathmandu: Jadibuti Association of Nepal (JABAN).

Singh, A.G., Kumar, A,. and Tewari, D.D. (2012). An ethnobotanical survey of medicinal plants used in Terai forest of western Nepal. J Ethnobiol Ethnomed 8: 19.

Uprety, Y., Asselin, H., Boon, E.K., Yadav, S., and Shrestha, K.K. (2010). Indigenous use and bio-efficacy of medicinal plants in the Rasuwa District, Central Nepal. Journal of Ethnobiology and Ethnomedicine 6:3, 1-10.

WHO. (2011). The World Traditional Medicines Situation in Traditional medicines: Global Situation, Issues and Challenges. Geneva: WHO (World Health Organization).

WHO. (2002). WHO Traditional Medicine Strategy. Geneva: World Health Organization (WHO). 